\title{
Circ_0026416 downregulation blocks the development of colorectal cancer through depleting MYO6 expression by enriching miR-545-3p
}

Lei Zhang ${ }^{1}$, Ranran Yu ${ }^{2}$, Chunhua Li ${ }^{1}$, Yu Dang ${ }^{1}$, Xiaoyu Yi ${ }^{1}$ and Lei Wang ${ }^{3 *}$

\begin{abstract}
Background: Emerging evidence reveals that the initiation and development of human cancers, including colorectal cancer (CRC), are associated with the deregulation of circular RNAs (circRNAs). Our study intended to disclose the role of circ_0026416 in the malignant behaviors of CRC.

Methods: The detection for circ_0026416 expression, miR-545-3p expression, and myosin VI (MYO6) mRNA expression was performed using quantitative real-time PCR (qPCR). CCK-8 assay, colony formation assay, transwell assay, and flow cytometry assay were applied for functional analysis to monitor cell proliferation, migration, invasion, and apoptosis. The protein levels of MYO6 and epithelial mesenchymal-transition (EMT) markers were detected by western blot. Mouse models were used to determine the role of circ_0026416 in vivo. The potential relationship between miR-545-3p and circ_0026416 or MYO6 was verified by dual-luciferase reporter assay and RIP assay.

Results: The expression of circ_0026416 was increased in CRC tumor tissues and cell lines. Circ_0026416 downregulation inhibited CRC cell proliferation, colony formation, migration, invasion, and EMT but induced cell apoptosis in vitro, and circ_0026416 knockdown also blocked tumor growth in vivo. MiR-545-3p was a target of circ_0026416, and rescue experiments indicated that circ_0026416 knockdown blocked CRC development by enriching miR545-3p. In addition, miR-545-3p targeted MYO6 and inhibited MYO6 expression. MiR-545-3p enrichment suppressed CRC cell malignant behaviors by sequestering MYO6. Importantly, circ_0026416 knockdown depleted MYO6 expression by enriching miR-545-3p.
\end{abstract}

Conclusion: Circ_0026416 downregulation blocked the development of CRC through depleting MYO6 expression by enriching miR-545-3p.

Highlights: 1. Circ_0026416 downregulation inhibits CRC development in vitro and in vivo.

2. Circ_0026416 regulates the expression of MYO6 by targeting miR-545-3p.

3. Circ_0026416 governs the miR-545-3p/MYO6 axis to regulate CRC progression.

\footnotetext{
*Correspondence: chinawanglei888@163.com

${ }^{3}$ Cancer Center, The Second Affiliated Hospital of Shandong University

of Traditional Chinese Medicine, No.1 Jingba Road, Shizhong District, Jinan City 250001, Shandong Province, China

Full list of author information is available at the end of the article
}

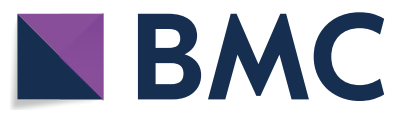

The Author(s) 2021. Open Access This article is licensed under a Creative Commons Attribution 4.0 International License, which permits use, sharing, adaptation, distribution and reproduction in any medium or format, as long as you give appropriate credit to the original author(s) and the source, provide a link to the Creative Commons licence, and indicate if changes were made. The images or other third party material in this article are included in the article's Creative Commons licence, unless indicated otherwise in a credit line to the material. If material is not included in the article's Creative Commons licence and your intended use is not permitted by statutory regulation or exceeds the permitted use, you will need to obtain permission directly from the copyright holder. To view a copy of this licence, visit http://creativecommons.org/licenses/by/4.0/. The Creative Commons Public Domain Dedication waiver (http://creativeco mmons.org/publicdomain/zero/1.0/) applies to the data made available in this article, unless otherwise stated in a credit line to the data. 
Keywords: circ_0026416, Colorectal cancer, miR-545-3p, MYO6

\section{Introduction}

Colorectal cancer (CRC) has an increasing incidence in recent years, mainly due to poor diet, poor lifestyle, smoking, and physical inactivity [1]. CRC has been the third most common cancer in 2020 , accounting for $10 \%$ of all cancer cases, and the second cause of cancer-related death [2]. A better understanding of CRC genomics has recently emerged, with promising therapeutic implications [3]. Emerging evidence suggests that the development of high-precision non-invasive screening tests using biomarkers to predict the risk of CRC or the early presence may contribute to the diagnosis and clinical practice of cancer, such as programmed cell death protein 1 (PD1) and $\mathrm{Na}+/ \mathrm{Ca} 2+$ exchangers (NCXs) [3-6]. The etiology of CRC is complex, and further research is needed to elucidate the pathogenesis of $\mathrm{CRC}$ from various perspectives to improve the outcomes of CRC treatment.

Circular RNAs (circRNAs) are covalently closed noncoding RNA molecules, deriving from a canonical splicing mechanism [7]. CircRNAs display high extent of tissue specificity and high stability compared to linear molecules [8]. Accordingly, circRNAs are regarded as promising non-invasive biomarkers of human cancers [9]. CircRNAs have been confirmed to be involved in various signaling pathways that are crucial in human diseases [10]. Besides, circRNAs are key regulators in cancer cell proliferation, motility, apoptosis, and angiogenesis [8]. In CRC, numerous circRNAs have been identified to be deregulated, and their functions have been partly clarified [11-13]. For example, circ_0053277 was overexpressed in CRC, and its knockdown inhibited CRC cell proliferation and migration [11]. Circ_0006401 displayed high expression in CRC, and its knockdown blocked tumor cell proliferation and liver metastasis in vivo [12]. Nonetheless, there are still numerous circRNAs with unclear functions. To date, numerous differentially expressed genes (DEGs) can be acquired from Gene Expression Omnibus (GEO) database or TCGA database, and various models used for the prognostic prediction of CRC were established based on these DEGs [14-18]. A dataset (GSE77661) from GEO database provides numerous circRNAs with aberrant expression in diverse cancer tissues. Among these circRNAs, circ_0026416 was shown to have aberrantly upregulated in CRC tissues. It is of great importance to explore the role of circ_0026416 in CRC, and the molecular mechanism of circ_0026416 in CRC needs further investigation.

The molecular sponge effects of circRNA on microRNAs (miRNAs) are widely demonstrated to illustrate the functional mode of circRNAs in biological processes [19]. For example, miR-132-3p was a tumor suppressor in CRC, and circ_0020397 aggravated CRC cell malignant properties via acting as miR-132-3p sponge to sequester miR-132-3p expression [20]. By bioinformatics analysis, we screened the target miRNAs of circ_0026416 and found that miR-545-3p was previously shown to inhibit cell proliferation in CRC [21]. Therefore, we determined the relationship between circ_0026416 and miR-545-3p in CRC development. MiRNAs are well-recognized to regulate gene expression by binding to gene $3^{\prime}$ untranslated region ( $\left.3^{\prime} \mathrm{UTR}\right)$ [22, 23]. Myosin VI (MYO6) was predicted as a target gene of miR-545-3p, and the oncogenic effect of MYO6 in CRC was widely documented $[24,25]$. Thus, we explored the interaction between miR545-3p and MYO6 in CRC to further disclose the functional mechanism of circ_0026416 in CRC.

The present study investigated the detailed functions of circ_0026416 in CRC cells and mouse models in vivo. Importantly, this study proposed a new network, circ_0026416/miR-545-3p/MYO6, to partly clarify the mechanism of circ_0026416 function in CRC, aiming to provide a theoretical basis for circ_0026416 as a biomarker in CRC.

\section{Materials and methods \\ Specimen collection}

Tumor tissues and corresponding normal (non-tumor) tissues were collected from a total of 43 CRC patients who underwent surgery at the Second Affiliated Hospital of Shandong University of Traditional Chinese Medicine. Patients never received any preoperative treatments. The characteristics of tumor tissues and normal tissues were identified by histopathology, and the clinicopathologic characteristics of these patients and the correlation between circ_0026416 expression and these characteristics were shown in Table 1. The collection of specimens was approved by all patients with written informed consent. Specimens were frozen in liquid nitrogen and placed in $-80^{\circ} \mathrm{C}$ conditions. This study was permitted by the Ethics Committee of the Second Affiliated Hospital of Shandong University of Traditional Chinese Medicine.

\section{Cell lines}

HCT116 (Bena, Beijing, China), DLD-1 (Bena), and HCT-8 (Bena) were cultured in RPMI-1640 medium (GIBCO, Grand Island, NY, USA) supplemented with $10 \%$ FBS. SW480 (Bena) was cultured in DMEM (GIBCO) containing 10\% FBS. Human normal colonic 
Table 1 Association between circ_0026416 expression and clinical clinicopathological parameters of CRC

\begin{tabular}{|c|c|c|c|c|}
\hline \multirow[t]{2}{*}{ Parameter } & \multirow{2}{*}{$\begin{array}{l}\text { Total } \\
\text { case }(n= \\
43)\end{array}$} & \multicolumn{2}{|c|}{ Circ_0026416 expression } & \multirow[t]{2}{*}{$P$ value ${ }^{a}$} \\
\hline & & $\operatorname{Low}(n=21)$ & High $(n=22)$ & \\
\hline Age (years) & & & & 0.4503 \\
\hline$\leq 60$ & 20 & 11 & 9 & \\
\hline$>60$ & 23 & 10 & 13 & \\
\hline Gender & & & & 0.6497 \\
\hline Female & 22 & 10 & 12 & \\
\hline Male & 21 & 11 & 10 & \\
\hline Tumor size & & & & $0.0003^{*}$ \\
\hline$\leq 5 \mathrm{~cm}$ & 20 & 16 & 4 & \\
\hline$>5 \mathrm{~cm}$ & 23 & 6 & 17 & \\
\hline TNM stages & & & & $0.0014^{*}$ \\
\hline$|-| \mid$ & 20 & 15 & 5 & \\
\hline III-IV & 23 & 6 & 17 & \\
\hline $\begin{array}{l}\text { Lymphatic metas- } \\
\text { tasis }\end{array}$ & & & & $<0.0001^{*}$ \\
\hline Negative & 20 & 17 & 3 & \\
\hline Positive & 23 & 4 & 19 & \\
\hline
\end{tabular}

CRC Colorectal cancer, TNM tumor-node-metastasis; ${ }^{*} P<0.05$; ${ }^{a}$ Chi-square test

epithelial cell (NCM460; Bluefbio, Shanghai, China), used as a non-cancer control, was cultured in DMEM plus $10 \%$ FBS. Human umbilical vein endothelial cells (HUVECs; Bena) were cultured in Ham's F-12 K medium (GIBCO) containing $10 \%$ FBS. These cells were maintained in a cell incubator at $37^{\circ} \mathrm{C}$ supplied with $5 \% \mathrm{CO}_{2}$.

\section{Quantitative real-time PCR (qPCR)}

Trizol reagent (Solarbio, Beijing, China) was utilized for total RNA isolation. For cDNA synthesis, a kit from Vazyme (Nanjing, China) named HiScript III 1st Strand cDNA Synthesis Kit, and a kit named miRNA 1st Strand cDNA Synthesis Kit (Vazyme) were applied using total RNA. Subsequently, AceQ Universal SYBR qPCR Master Mix (Vazyme) was used for qPCR reaction. Relative expression was normalized using the $2^{-\Delta \Delta \mathrm{Ct}}$ method, with GAPDH (for circRNA and mRNA) or U6 (for miRNA) as the internal reference. The sequences of primers were exhibited below:

circ_0026416, F 5'-GATGAATGTCAAGCTGGC CC- $3^{\prime}$, and $\mathrm{R}$ 5'-TCATGTAGGCAGCATCCACA-3'; KRT6C, F 5'-CTTCCCTGCTCTCCGAGGTA-3', and R 5'-TGCTCAGATGGGGCAGGTAT-3'; miR-545-3p, F 5'-GCGCGTCAGCAAACATTTATT-3', and R 5'-AGT GCAGGGTCCGAGGTATT-3'; MYO6, F 5'-AGAATC AGGAGCCGGCAAAA-3', and $\mathrm{R}$ 5'-CCAAAGGCT TCTAGGAGTGGG-3'; GAPDH, F 5'-ACAGCCTCA AGATCATCAGC- $3^{\prime}$, and R 5'-GGTCATGAGTCC TTCCACGAT-3'; U6, F 5'-CTCGCTTCGGCAGCA
CATATACT- $3^{\prime}$ and $\mathrm{R}$ 5'-ACGCTTCACGAATTTGCG TGTC-3'.

\section{RNase $R$ digestion and ActD treatment}

To ensure the existence of circ_0026416, total RNA was

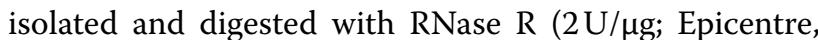
Madison, WI, USA) for $30 \mathrm{~min}$ at $37^{\circ} \mathrm{C}$ and next used for qPCR.

To check the stability of circ_0026416, Actinomycin D $(10 \mu \mathrm{g} / \mathrm{mL}$; Sigma-Aldrich, St. Louis, MO, USA) was added into culture medium to culture cells for the indicated time $(0,4,8,12$, or $24 \mathrm{~h})$. RNA isolation was performed using these cells at different time, and subsequent qPCR was conducted.

\section{Cell transfection}

Lipofectamine 3000 reagent (Invitrogen, Carlsbad, CA, USA) was used for cell transfection. Simply put, cells were plated into 6 -well plates $\left(5 \times 10^{5}\right.$ cells/well $)$ and then transfected with oligonucleotides $(50 \mathrm{nM})$ or vectors $(2 \mu \mathrm{g})$. Small interference RNA (siRNA) targeting circ_0026416 (si-circ_0026416) and its negative control (si-NC) were synthesized by Genepharma (Shanghai, China). MiR-545-3p mimic (miR-545-3p) and miR-545-3p inhibitor (anti-miR-545-3p) and their negative controls (miR-NC and anti-miR-NC) were provided by Ribobio (Guangzhou, China). MYO6 overexpression vector (pcDNA-MYO6) and its blank control (pcDNAcon) were constructed by Genepharma.

\section{CCK-8 assay}

After transfection, cells were plated in 96-well plates (5000 cells/well), with one sample in triplicate. Then, cells were continued to culture. CCK- 8 reagent was used to treat cells at $0,24,48$, or $72 \mathrm{~h}$ for $2 \mathrm{~h}$. Subsequently, the absorbance at $450 \mathrm{~nm}$ was measured using a microplate reader (BioTek, Winooski, Vermont, USA).

\section{Colony formation assay}

After transfection, cells were plated in 6-well plates (200 cells/well). Cells were cultured in $37^{\circ} \mathrm{C}$ incubator containing $5 \% \mathrm{CO}_{2}$ for 12 days. The growth of colonies was observed every day. The surface of cell colonies was washed by PBS, fixed by methanol and stained with crystal violet. The number of colonies was observed using a microscope (Leika, Wetzlar, Germany).

\section{Transwell assay}

Transwell chambers coated with or without Matrigel (BD Biosciences, Franklin Lakes, NJ, USA) were used for cell invasion or cell migration analysis. At $24 \mathrm{~h}$ post-transfection, cells were collected and resuspended in serumdepleted culture medium. Then, cells were transferred to 
the top of chambers, with culture medium supplemented with 10\% FBS in the bottom of chambers. Cells in chambers were cultured for $24 \mathrm{~h}$ to induce invasion or migration. Next, cells in the lower surface of chambers were fixed with methanol and stained with crystal violet. The magnification of $100 \times$ was used to capture the images of migration or invaded cells under a microscope.

\section{Tube formation assay}

Ninety-six-well plates were coated with Matrigel $(60 \mu \mathrm{L} /$ well) and maintained at $37^{\circ} \mathrm{C}$ incubator for $1 \mathrm{~h}$. At $48 \mathrm{~h}$ post-transfection, tumor-conditioned medium (tumor supernatant: DMEM: FBS $=4: 5: 1$ ) was prepared. HUVECs were cultured with tumor-conditioned medium and HUVEC suspension for $6 \mathrm{~h}$. Five randomly selected fields were used to capture images of tube using an inverted microscope (magnification, $\times 40$ ).

\section{Flow cytometry assay}

At $48 \mathrm{~h}$ post-transfection, the Annexin V-FITC Apoptosis Detection Kit (Beyotime, Shanghai, China) was used to monitor cell apoptosis using a flow cytometer (BD Biosciences) according to the protocol from kit.

\section{Western blot}

Western blot was carried out as previously mentioned [24]. All antibodies were obtained from Abcam (Cambridge, MA, USA), including anti-E-Cadherin (ab40772; dilution: 1/10,000), anti-vimentin (ab92547; dilution: 1/2,000), anti-N-Cadherin (ab76011; dilution: 1/10,000), anti-MYO6 (ab230478; dilution: 1/1,000), and goat-anti rabbit secondary antibody (ab205718; dilution: 1/20,000).

\section{Xenograft model}

Lentivirus-packaged shRNA targeting circ_0026416 (sh-circ_0026416) and sh-NC were provided by Genepharma. Nude mice (female, 6-week-old) were purchased from Vital River Animal Technology Co., Ltd. (Beijing, China) and divided into 2 groups ( $n=6$ per group). SW480 cells were infected with lentivirus-packaged shcirc_0026416 or sh-NC and subcutaneously injected into nude mice $\left(2 \times 10^{6}\right.$ cells/mouse). The condition of mice was observed every day. Tumor volume (length $\times$ width $^{2}$ $\times 0.5$ ) was recorded once a week. After 4 weeks, all mice were euthanized to remove tumor tissues. The study of animal was approved by the Animal Care and Use Committee of the Second Affiliated Hospital of Shandong University of Traditional Chinese Medicine.

\section{Dual-luciferase reporter assay}

The binding site between circ_0026416 and miR-545-3p was provided by circRNA interactome (https://circi nteractome.nia.nih.gov/). The binding site between
miR-545-3p and MYO6 3'UTR was provided by Targetscan (http://www.targetscan.org/vert_72/). The mutant-type sequences of circ_0026416 and MYO6 $3^{\prime}$ UTR (mutation at miR-545-3p binding site) were synthesized. Reporter plasmids, including circ_0026416 WT, circ_0026416 MUT, MYO6 3'UTR WT and MYO6 3'UTR MUT, were constructed into pmirGLO vector (Promega, Madison, WI, USA). HCT-8 and SW480 cells were transfected with miR-545-3p or miR-NC and reporter plasmid and next incubated for $48 \mathrm{~h}$. Cells were collected and used for luciferase activity analysis using the Dual-Luciferase Reporter Assay System (Promega).

\section{RIP assay}

Magna RIP ${ }^{\mathrm{TM}}$ Kit (Millipore, Billerica, MA, USA) was utilized for RIP assay. In brief, cells were lysed using RIP lysis solution, and cell lysates were incubated with protein A/G magnetic beads conjugated with Ago2 antibody (Millipore) or IgG antibody (Millipore). RNA complexes bound to beads were eluted and analyzed by qPCR.

\section{Statistical analysis}

Data were collected from at least three separate experiments. The results were expressed as the mean \pm standard deviation (SD). Differences in various groups were assessed by Student's $t$-test or analysis of variance. Statistical analysis was conducted using GraphPad Prism 6 (GraphPad Software, San Diego, USA), and figures were subsequently generated. $P$ less than 0.05 was considered statistically significant.

\section{Results}

The expression of circ_0026416 was increased in CRC tumor tissues and cells

The clinical data in Table 1 displayed that high circ_0026416 expression was significantly associated with large tumor size, advanced TNM stages, and positive lymphatic metastasis. We examined the expression of circ_0026416 in clinical specimens and found that circ_0026416 expression was markedly enhanced in tumor tissues compared to normal tissues (Fig. 1A). Besides, circ_0026416 expression was also increased in CRC cell lines, including HCT-116, DLD-1, HCT8 , and SW480 cells, particularly in HCT-8 and SW480 cells (Fig. 1B). HCT-8 and SW480 cells were thus selected in the following assays. For the identification of circ_0026416 existence and stability, we found that circ_0026416 was resistant to RNase R digestion, while KRT6C mRNA was notably digested by RNase R (Fig. $1 \mathrm{C}$ and D). In addition, after ActD treatment, the expression of circ_0026416 was hardly declined, while the expression of KRT6C mRNA was markedly reduced (Fig. 1E, F). These data verified the existence and stability 

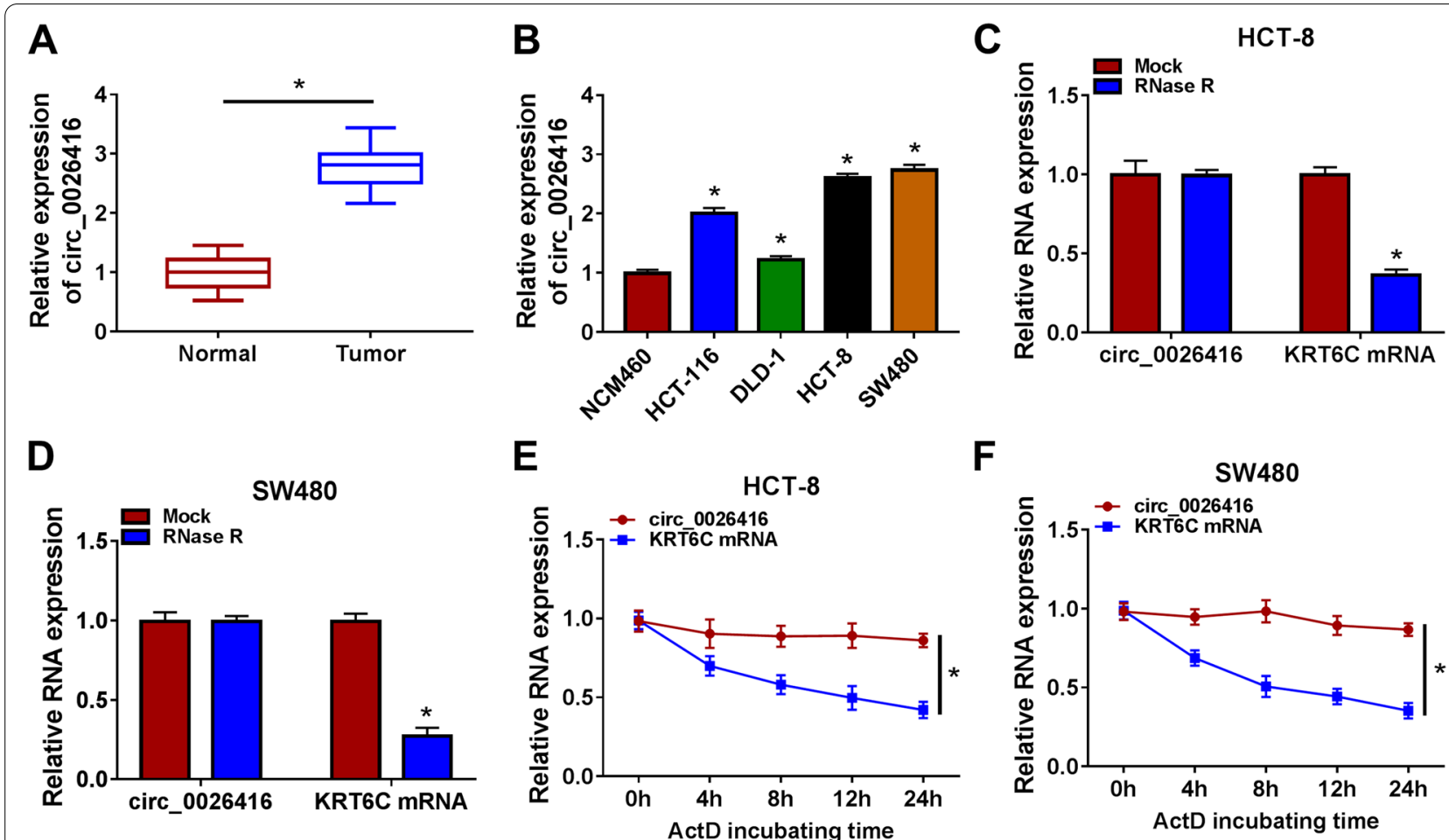

Fig. 1 Circ_0026416 was upregulated in CRC, with high stability. A Circ_0026416 expression in clinical specimens was checked by qPCR. B Circ_0026416 expression in CRC cell lines and non-cancer cell line was checked by qPCR. C, D The existence of circ_0026416 was identified using RNase R. E, F The stability of circ_0026416 was identified using ActD. ${ }^{*} P<0.05$

of circ_0026416 and presented the high expression of circ_0026416 in CRC tissues and cells.

\section{Circ_0026416 downregulation suppressed CRC cell malignant behaviors and solid tumor growth in vivo} HCT-8 and SW480 cells were transfected with sicirc_0026416 or si-NC for functional analysis. Three different si-circ_0026416 oligonucleotides were synthesized to mediate circ_0026416 knockdown. The expression of circ_0026416 was strikingly decreased in HCT-8 and SW480 cells after si-circ_0026416 (or si-circ_0026416\#1, si-circ_0026416\#2) transfection (Fig. 2A; Figure S1A). In function, circ_0026416 downregulation suppressed the proliferative capacity and colony formation ability of HCT- 8 and SW 480 cells by CCK- 8 and colony formation assays (Fig. 2B-D; Figure S1B-D). Transwell assay indicated that the migration and invasion abilities were markedly suppressed in HCT-8 and SW480 cells transfected with si-circ_0026416 (Fig. 2E, F; Figure S1E and F). Tube formation assay showed that the downregulation of circ_0026416 inhibited the number of branches of tubes, thus inhibiting angiogenesis (Fig. 2G; Figure $\mathrm{S} 1 \mathrm{G})$. Flow cytometry assay revealed that the apoptotic rate of HCT-8 and SW480 cells transfected with sicirc_0026416 was strikingly promoted (Fig. 2H; Figure
S1H). Moreover, EMT-related markers were also quantified by western blot. The data showed that the level of E-Cadherin was enhanced, while the levels of vimentin and N-Cadherin were reduced in HCT-8 and SW480 cells transfected with si-circ_0026416 (Fig. 2I, J; Figure S1I and J). Animal study was further conducted to determine the role of circ_0026416 in vivo. The data presented that circ_0026416 knockdown in SW480 cells markedly depleted tumor volume and tumor weight (Fig. $2 \mathrm{~K}-\mathrm{M}$ ). The expression of circ_0026416 was strikingly decreased in sh-circ_0026416-treated tumor tissues (Fig. 2N). These data indicated that circ_0026416 downregulation suppressed CRC cell malignant behaviors and solid tumor growth in vivo.

\section{MiR-545-3p was a target of circ_0026416}

Bioinformatics tool circular RNA interactome showed the binding site between circ_0026416 and miR-545-3p sequence fragments (Fig. 3A). The expression of miR$545-3 p$ was notably increased in HCT-8 and SW480 cells transfected with miR-545-3p mimic compared to miR-NC (Fig. 3B). Dual-luciferase reporter assay presented that miR-545-3p transfected with circ_0026416 WT significantly reduced luciferase activity in HCT-8 and SW480 cells (Fig. 3C, D). RIP assay presented that 


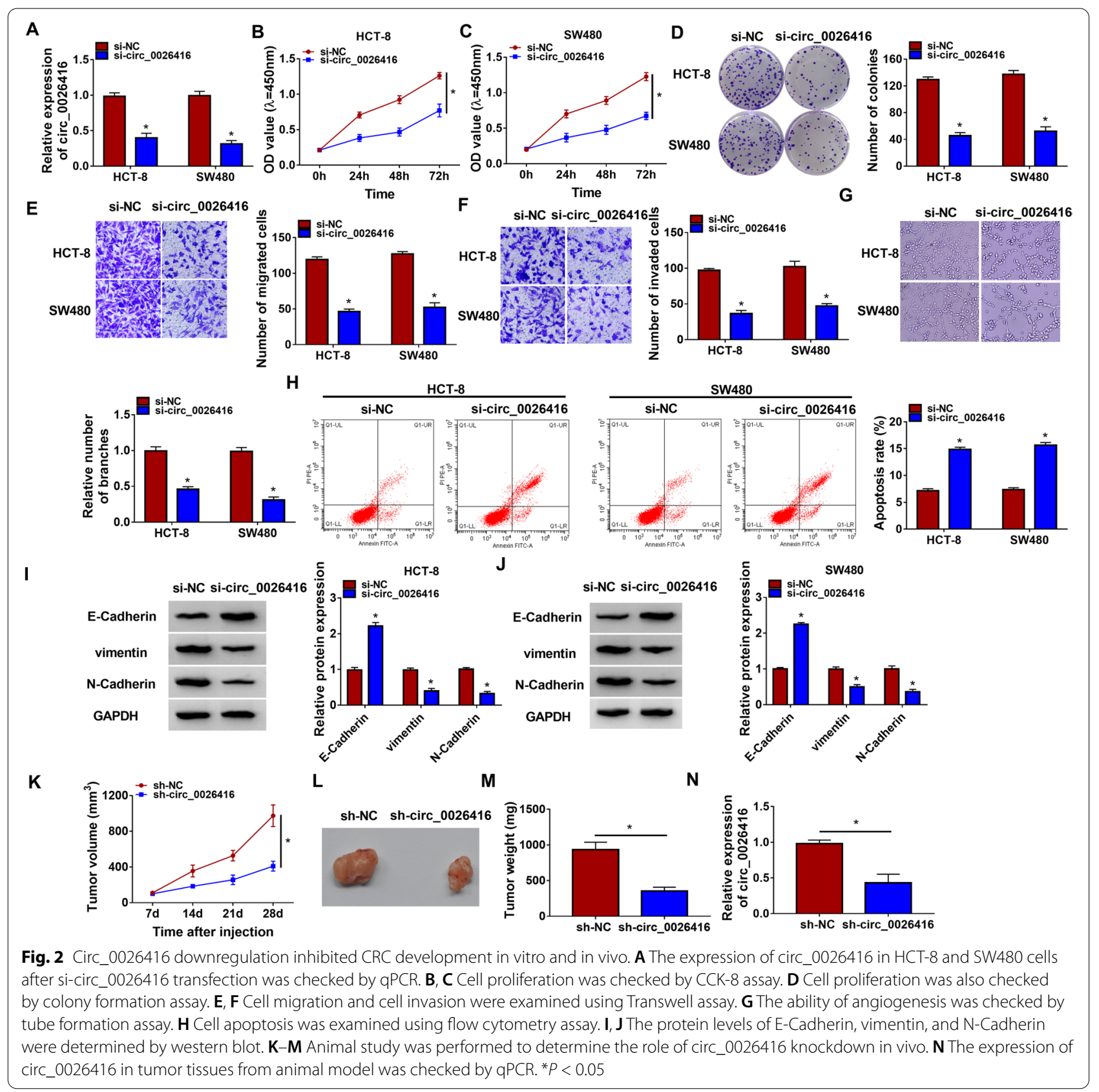

circ_0026416 and miR-545-3p were abundantly enriched in anti-Ago2 RIP group compared to anti-IgG RIP group (Fig. 3E, F). The expression of miR-545-3p was remarkably downregulated in tumor tissues and cell lines of CRC (Fig. 3G, H). Besides, miR-545-3p expression in tumor tissues was negatively correlated with circ_0026416 expression $(r=-0.5758, P<0.0001$; Figure S2). The expression of miR-545-3p was strikingly decreased in HCT-8 and SW480 cells transfected with anti-miR545-3p (Fig. 3I). Moreover, miR-545-3p expression was markedly increased in HCT-8 and SW480 cells transfected with si-circ_0026416 but partly repressed in HCT-8 and SW480 cells transfected with si-circ_0026416 + anti-miR-545-3p (Fig. 3J, K).

\section{Circ_0026416 downregulation suppressed CRC cell malignant behaviors by enriching miR-545-3p}

Function rescue experiments were performed to determine the relationship between circ_0026416 and miR-545-3p. CCK-8 assay and colony formation assay 


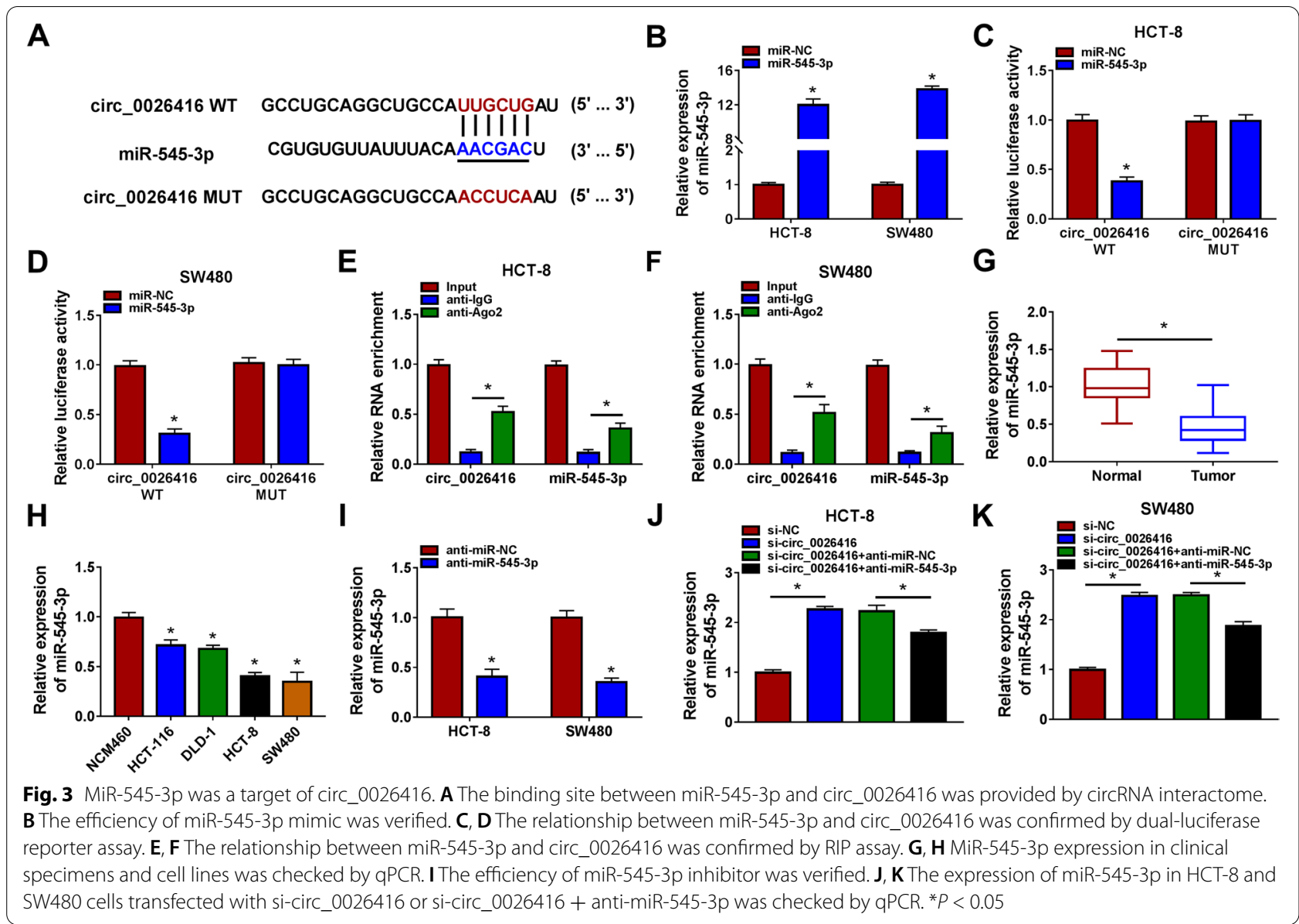

presented that cell proliferation and colony formation were suppressed in HCT-8 and SW480 cells transfected with si-circ_0026416 but partly recovered in cells transfected with si-circ_0026416 + anti-miR-545-3p (Fig. 4A-C). Transwell assay manifested that cell migration and cell invasion were blocked in HCT-8 and SW480 cells after circ_0026416 knockdown, and further miR545-3p downregulation partly enhanced cell migration and invasion (Fig. 4D, E). Tube formation assay revealed that the inhibition of miR-545-3p promoted angiogenesis that was inhibited by circ_0026416 knockdown alone (Fig. 4F). In addition, circ_0026416 knockdown-induced HCT-8 and SW480 cell apoptosis was largely alleviated by miR-545-3p knockdown (Fig. 4G). Moreover, E-Cadherin level promoted in HCT-8 and SW480 cells transfected with si-circ_0026416 was partly repressed in HCT-8 and SW 480 cells transfected with si-circ_0026416 + anti-miR-545-3p, while vimentin and N-Cadherin levels suppressed in HCT-8 and SW480 cells transfected with si-circ_0026416 were partly recovered in HCT-8 and SW480 cells transfected with si-circ_0026416 + anti-miR-545-3p (Fig. 4H, I). The data indicated that circ_0026416 downregulation suppressed CRC cell malignant behaviors by enriching miR-545-3p.

\section{MiR-545-3p suppressed MYO6 expression}

Bioinformatics tool Targetscan showed the binding site between miR-545-3p and MYO6 3'UTR, implying that MYO6 was a potential target of miR-545-3p (Fig. 5A). Dual-luciferase reporter assay showed that miR-545-3p transfected with MYO6 3'UTR significantly reduced luciferase activity in HCT-8 and SW480 cells (Fig. 5B, C). RIP assay displayed that miR-545-3p and MYO6 were markedly enriched in the anti-Ago2 RIP group compared to anti-IgG RIP group (Fig. 5D, E). The expression of MYO6 mRNA was notably reinforced in tumor tissues (Fig. 5F), and the protein level of MYO6 was also notably reinforced in 6 randomly selected tumor tissues from CRC patients (Fig. 5G). The protein level of MYO6 was markedly enhanced in CRC cell lines (Fig. 5H). In addition, the protein level of MYO6 was largely increased in HCT-8 and SW480 cells after pcDNA-MYO6 transfection (Fig. 5I). The protein level of MYO6 was significantly declined in HCT-8 


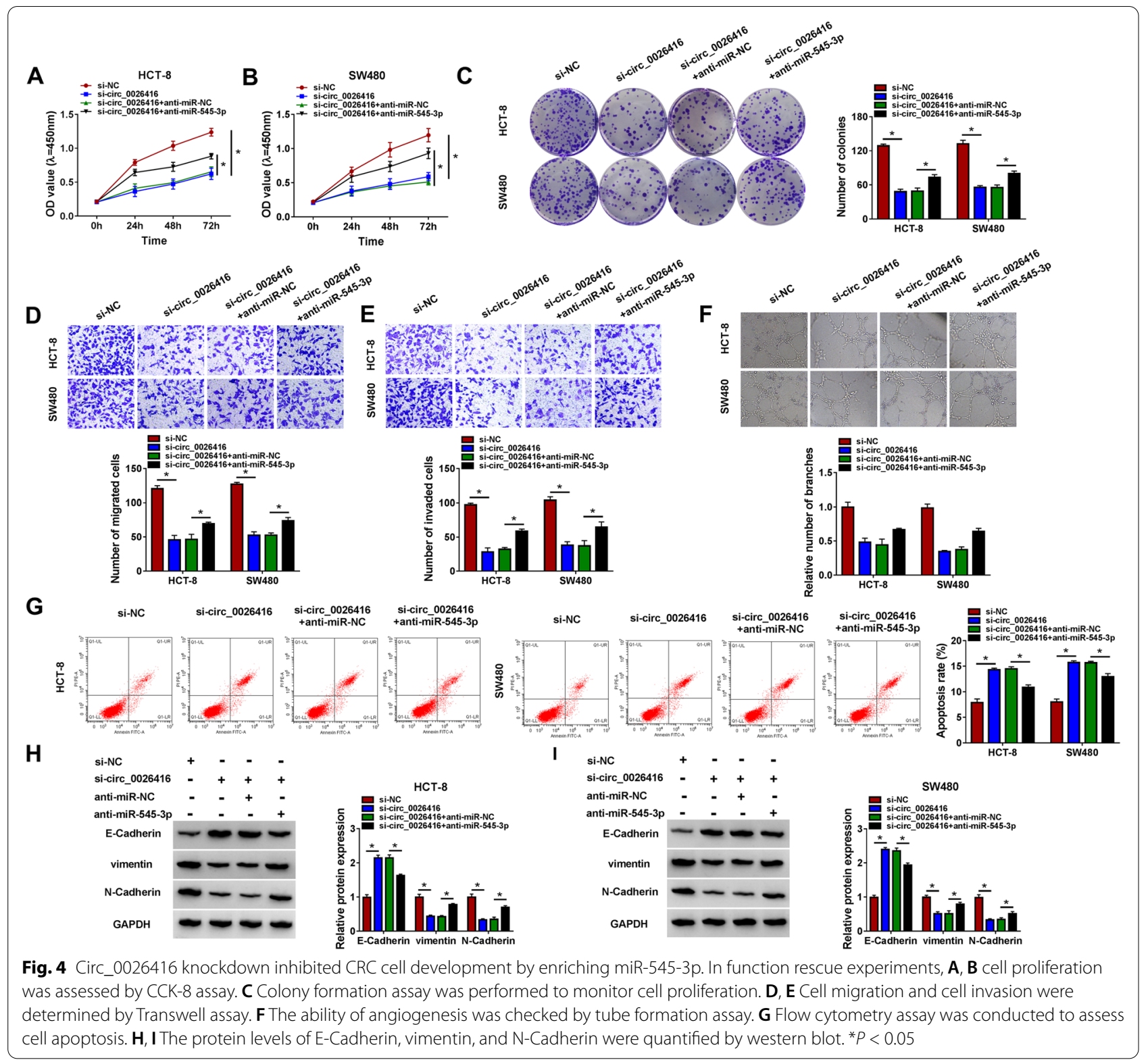

and SW480 cells transfected with miR-545-3p alone but recovered in cells transfected with miR-545-3p + pcDNA-MYO6 (Fig. 5J). The data mainly indicated that miR-545-3p targeted MYO6 and inhibited MYO6 expression.

\section{MiR-545-3p restoration inhibited CRC cell malignant behaviors by inhibiting MYO6 expression}

CCK- 8 assay and colony formation assay manifested that cell proliferative capacity and colony formation ability were notably inhibited in HCT-8 and SW480 cells transfected with miR-545-3p alone but partly recovered in cells transfected with miR-545-3p + pcDNA-MYO6
(Fig. 6A-C). Transwell assay presented that the capacity of cell migration and cell invasion was notably sequestered in HCT- 8 and SW 480 cells transfected with miR545-3p alone but partly restored in cells transfected with miR-545-3p + pcDNA-MYO6 (Fig. 6D, E). Tube formation assay indicated that angiogenesis in HCT-8 and SW480 cells was inhibited by miR-545-3p restoration but partially recovered by MYO6 reintroduction (Fig. 6F). Flow cytometry assay revealed that miR-545-3p restoration-induced HCT-8 and SW480 cell apoptosis was relieved by the reintroduction of MYO6 (Fig. 6G). Additionally, the expression of E-Cadherin was increased, while the expression of vimentin and $\mathrm{N}$-Cadherin was 


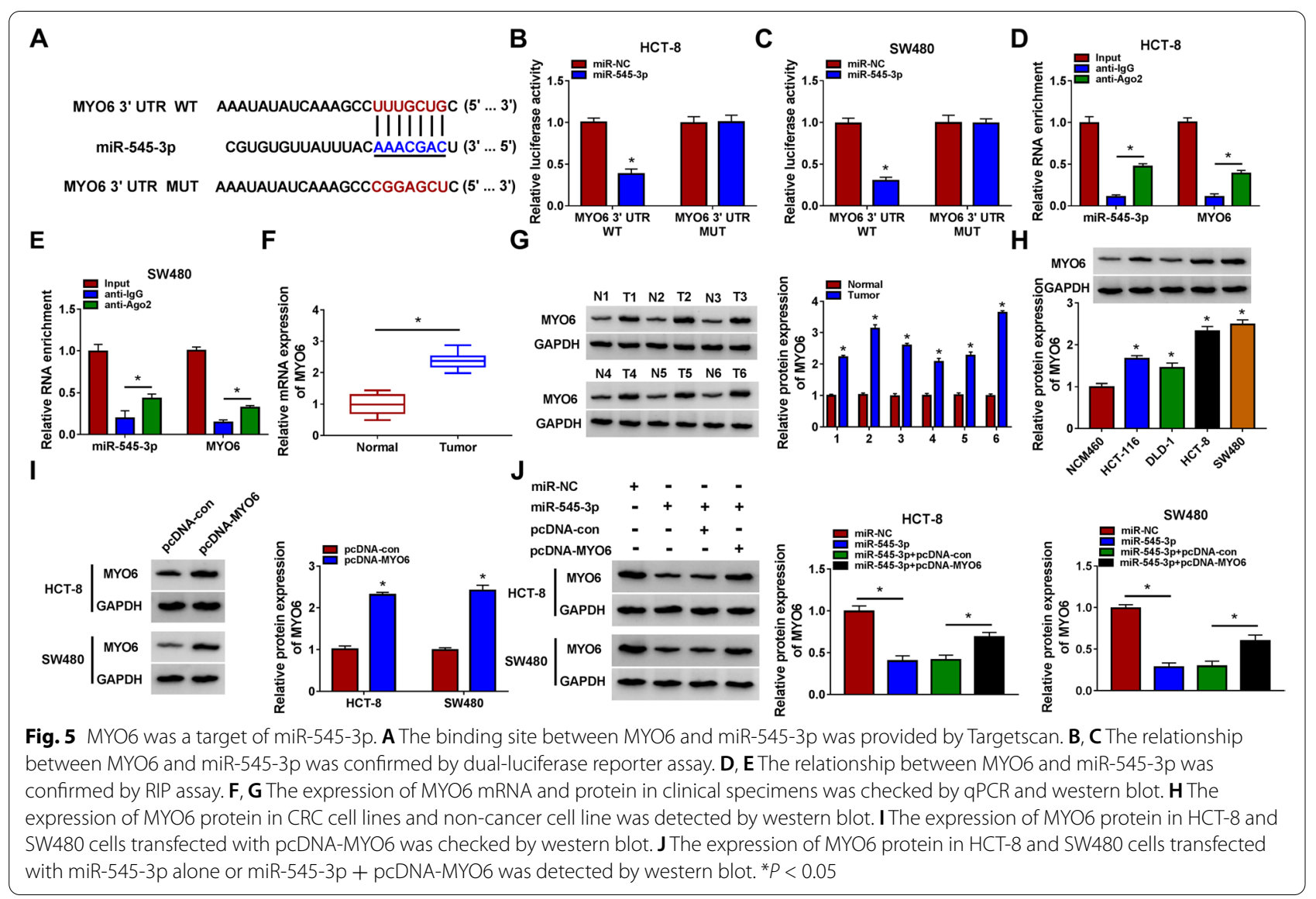

decreased in HCT-8 and SW480 cells transfected with miR-545-3p alone. However, the expression of E-Cadherin was partly repressed, while the expression of vimentin and N-Cadherin was restored in HCT-8 and SW480 cells transfected with miR-545-3p + pcDNAMYO6 (Fig. 6H, I). The data revealed that miR-545-3p restoration inhibited CRC cell malignant behaviors by inhibiting MYO6 expression.

\section{Circ_0026416 knockdown enriched miR-545-3p expression} and thus inhibited MYO6 expression

We further examined the expression of MYO6 mRNA and protein in HCT-8 and SW480 cells transfected with si-circ_0026416 alone or si-circ_0026416 + anti-miR545-3p. The data showed that the mRNA and protein levels of MYO6 were strikingly decreased in HCT-8 and SW480 cells transfected with si-circ_0026416 alone but largely recovered in cells transfected with si-circ_0026416 + anti-miR-545-3p (Fig. 7A-D). The data indicated that circ_0026416 knockdown enriched miR-545-3p expression and thus inhibited MYO6 expression.

\section{Discussion}

Recently, the function of newly identified circRNAs in CRC has been declared by an increasing number of literature [26-28], which contributes to the understanding of CRC pathogenesis. Our study exploited the function of circ_0026416 whose expression was strengthened in CRC tissues and cells and found that circ_0026416 silencing inhibited CRC cell proliferation, migration, invasion, angiogenesis, and survival. In terms of mechanism, we identified that circ_0026416 was involved in CRC development by regulating MYO6 expression via acting as miR-545-3p sponge. These results deepened our understanding of the role of circRNA in CRC progression.

Due to the advance of high-throughput RNA sequencing, a large number of circRNAs are considered to play important effects in cancer progression [29]. Based on this technology, circ_0026416 was identified to be markedly upregulated in CRC tumor tissues in GEO database (accession: GSE77661). A recent study reported that circ_0026416 expression was abnormally reinforced in tissues and plasma of CRC patients, and high circ_0026416 expression was linked to poor prognosis of CRC patients [30]. Further study manifested that the deficiency of circ_0026416 inhibited CRC cell proliferation, 


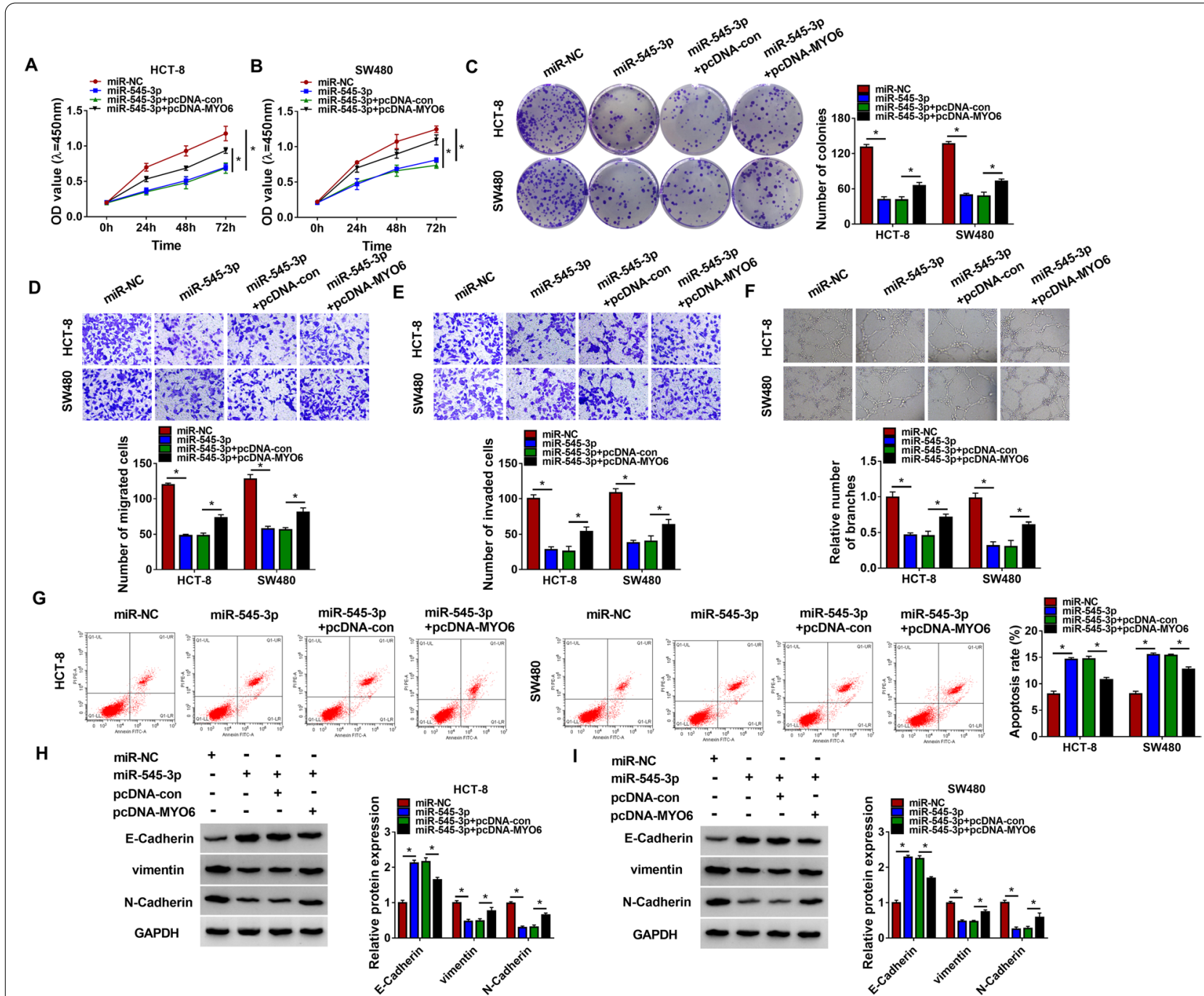

Fig. 6 MiR-545-3p restoration blocked CRC cell malignant behaviors by targeting MYO6. In function rescue experiments, A, B cell proliferation was assessed by CCK-8 assay. C Cell proliferation was assessed by colony formation assay. D, E Cell migration and cell invasion were examined using Transwell assay. $\mathbf{F}$ The ability of angiogenesis was checked by tube formation assay. $\mathbf{G}$ Flow cytometry assay was conducted to assess cell apoptosis. $\mathbf{H}, \mathbf{I}$ The protein levels of E-Cadherin, vimentin and $\mathrm{N}$-Cadherin were quantified by western blot. ${ }^{*} P<0.05$

invasion, and solid tumor growth in vivo [30]. Consistent with this study, our functional experiments displayed that circ_0026416 downregulation repressed the proliferation, migration, invasion, and angiogenesis of HCT-8 and SW480 cells and blocked solid tumor growth in animal models. These findings highlighted the cancer-promoting effects of circ_0026416 in CRC. At present, gene therapy is a promising treatment for cancer, and the delivery of siRNA-mediated oncogene suppression based on nanotechnology is a new strategy for CRC therapy [31, 32]. This study hinted that siRNA-mediated circ_0026416 inhibition might be a novel strategy to prevent the development of CRC.
To address the action mechanism of circ_0026416 in CRC, we identified miRNAs targeted by circ_0026416. MiR-545-3p attracted our interest because the role of miR-545-3p had been mentioned in CRC. For example, miR-545-3p overexpression inhibited CRC cell proliferation and colony formation, and its overexpression also impeded tumor growth in vivo [21]. Besides, miR-545-3p enrichment effectively induced CRC cell cycle arrest at G1 phase [33]. Our study discovered that miR-545-3p deficiency partly reversed the effects of circ_0026416 downregulation and recovered CRC cell malignant behaviors, while miR-545-3p restoration suppressed the growth, migration, invasion, and angiogenesis of CRC cells. Interestingly, miR-545-3p was also reported to 


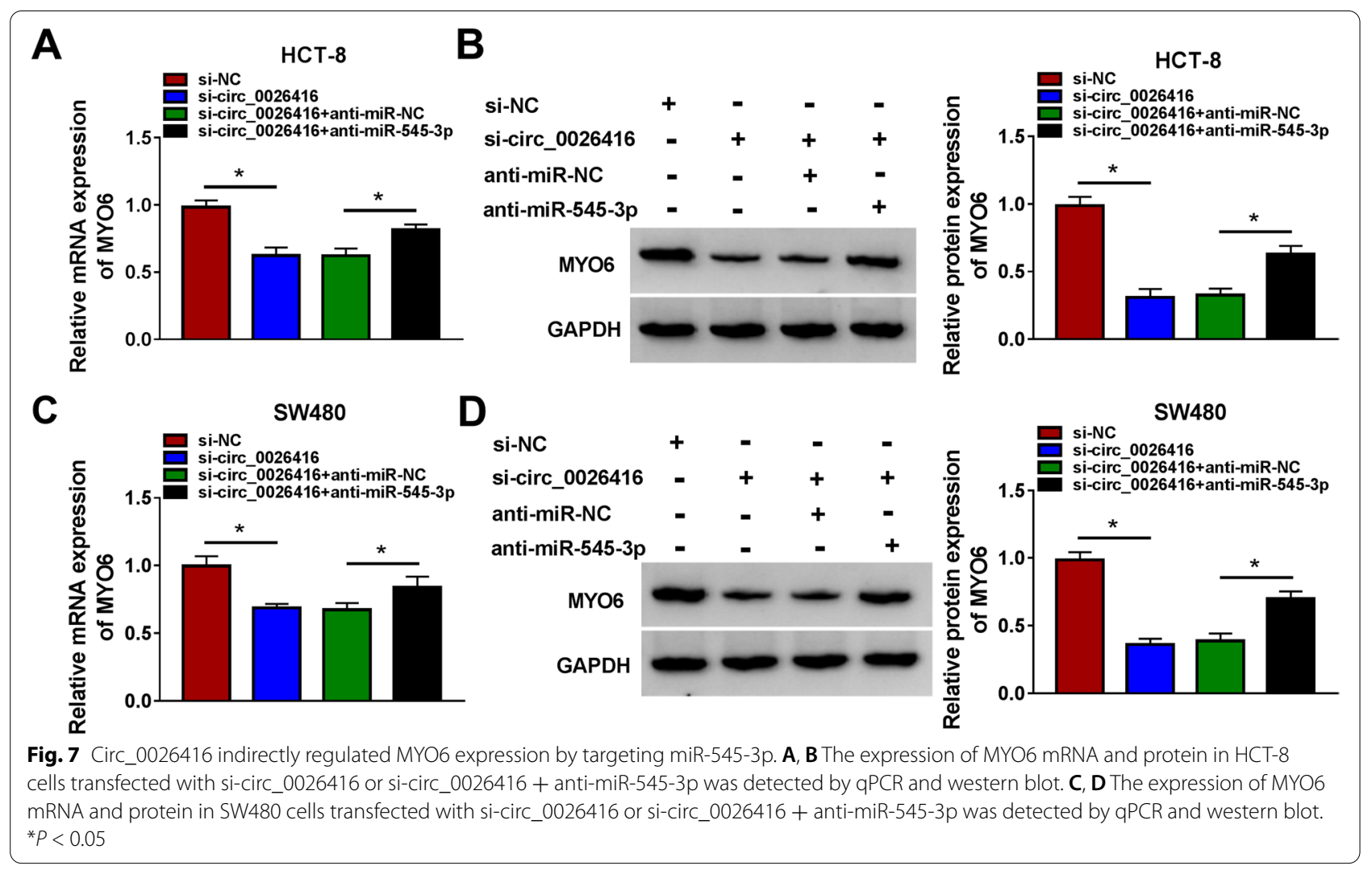

inhibit the progression of several other cancers, such as hepatocellular carcinoma and non-small cell lung cancer $[34,35]$, suggesting that miR-545-3p was a widelyacknowledged tumor suppressor in cancers. Here, partial restoration was observed in rescue experiments, possibly because miR-545-3p was only one of the targets of circ_0026416, and all of these targets functioned in circ_0026416-mediated regulatory networks.

Further analysis identified that miR-545-3p inhibited MYO6 expression by binding to MYO6 3'UTR. The reintroduction of MYO6 abolished the effects of miR-545-3p restoration and restored the malignant behaviors of CRC cells. MYO6 was an oncogene, which had been demonstrated in diverse cancers [36-38]. In CRC, the knockdown of MYO6 was shown to repress CRC cell growth and promote cell apoptosis [24]. Moreover, MYO6 was proposed to be involved in the circ_0000231/miR502-5p pathway in the regulation of CRC progression [39]. Similarly, we found that circ_0026416 downregulation reduced the expression of MYO6 via enriching the level of miR-545-3p; thus, the circ_0026416/miR-545-3p/ MYO6 regulatory network was established. Our study highlighted that oncogenic MYO6 was suppressed by miR-545-3p, while circ_0026416 acted as miR-545-3p sponge to alleviate the suppression of MYO6, thus promoting CRC carcinogenesis.
This study is only a preliminary study that illustrates the role of circ_0026416 in CRC, and the clinical application of circ_0026416 needs to be further exploited. Besides, the existing evidence published that some key regulators in CRC tumorigenesis, such as epiregulin (EREG) and amphiregulin (AREG), were differently expressed in right- and left-sided cases of CRC, and the mutation rate of some oncogenes or tumor suppressor genes was various in different sidedness of CRC [40]. This suggested that the expression pattern might be different in in rightand left-sided CRC. However, there was a lack of data on the sidedness of CRC, and we thus did not assess the difference of circRNA role in right- and left-sided cases. The future work should attach importance to these issues.

\section{Conclusion}

In summary, our study discovered that circ_0026416 downregulation inhibited CRC cell malignant behaviors in vitro and solid tumor growth in vivo. Importantly, we provided a new mechanism that circ_0026416 knockdown suppressed CRC development by depleting MYO6 via enriching miR-545-3p. We mainly defined that circ_0026416 was a carcinogenic driver in CRC, and circ_0026416 promoted CRC progression partly by targeting the miR-545-3p/MYO6 pathway. Further 
extensive in-depth studies are required to confirm these findings. All in all, this study hints that the inhibition of circ_0026416 mediated by siRNA may be a promising approach for CRC therapy.

\section{Abbreviations}

CRC: Colorectal cancer; circRNAs: Circular RNAs; siRNA: Small interference RNA; MYO6: Myosin VI; qPCR: Quantitative real-time PCR; EMT: Epithelial mesenchymal-transition.

\section{Supplementary Information}

The online version contains supplementary material available at https://doi. org/10.1186/s12957-021-02407-y.

Additional file 1: Figure S1. Circ 0026416 downregulation inhibited CRC cell malignant behaviors. (A) The expression of circ_0026416 in HCT-8 and SW480 cells after si-circ $0026416 \# 1$ or si-circ $0026416 \# 2$ transfection was checked by qPCR. In these transfected cells, (B-D) cell proliferation was checked by CCK-8 assay and colony formation assay. (E and F) Cell migration and cell invasion were examined using Transwell assay. (G) The ability of angiogenesis was checked by tube formation assay. (H) Cell apoptosis was examined using flow cytometry assay. (I and J) The protein levels of $\mathrm{E}$-Cadherin, vimentin and $\mathrm{N}$-Cadherin were determined by western blot. ${ }^{*} P<0.05$.

Additional file 2: Figure S2. MiR-545-3p expression was negatively correlated with circ_0026416 expression in tumor tissues.

\section{Acknowledgements}

None.

\section{Authors' contributions}

Ranran Yu and Chunhua Li designed and performed the research; Yu Dang, Xiaoyu Yi, and Lei Wang analyzed the data; Lei Zhang wrote the manuscript. All authors read and approved the final manuscript.

\section{Funding}

None.

\section{Availability of data and materials}

Not applicable.

\section{Declarations}

Ethics approval and consent to participate

Written informed consents were obtained from all participants and this study was permitted by the Ethics Committee of the Second Affiliated Hospital of Shandong University of Traditional Chinese Medicine.

\section{Consent for publication}

Not applicable.

\section{Competing interests}

The authors declare that they have no conflict of interest.

\section{Author details}

'Department of General Surgery, The Second Affiliated Hospital of Shandong University of Traditional Chinese Medicine, Jingba Road, Shizhong District, Jinan City 250001, Shandong Province, China. ${ }^{2}$ Department of Pathology, The Second Affiliated Hospital of Shandong University of Traditional Chinese Medicine, Jingba Road, Shizhong District, Jinan City 250001, Shandong Province, China. ${ }^{3}$ Cancer Center, The Second Affiliated Hospital of Shandong University of Traditional Chinese Medicine, No.1 Jingba Road, Shizhong District, Jinan City 250001, Shandong Province, China.
Received: 2 June 2021 Accepted: 21 September 2021

Published online: 14 October 2021

\section{References}

1. Kuipers EJ, et al. Colorectal cancer. Nat Rev Dis Primers. 2015;1:15065.

2. Sung H, et al. Global Cancer Statistics 2020: GLOBOCAN estimates of incidence and mortality worldwide for 36 cancers in 185 countries. CA Cancer J Clin. 2021;71(3):209-49.

3. Boussios $\mathrm{S}$, et al. The developing story of predictive biomarkers in colorectal cancer. J Pers Med. 2019;9(1):12.

4. Dickinson BT, et al. Molecular markers for colorectal cancer screening. Gut. 2015;64(9):1485-94.

5. Qu Y, et al. Construction of prognostic predictor by comprehensive analyzing alternative splicing events for colon adenocarcinoma. World J Surg Oncol. 2020;18(1):236.

6. Xia Z, Wang C, Zhang $\mathrm{H}$. The downregulation of NCXs is positively correlated with the prognosis of stage II-IV colon cancer. World J Surg Oncol. 2021;19(1):177.

7. Starke $S$, et al. Exon circularization requires canonical splice signals. Cell Rep. 2015;10(1):103-11.

8. Verduci L, et al. CircRNAs: role in human diseases and potential use as biomarkers. Cell Death Dis. 2021;12(5):468.

9. Chen $Y$, et al. Progress in research on the role of circular RNAs in lung cancer. World J Surg Oncol. 2018;16(1):215.

10. Li YF, Zhang J, Yu L. Circular RNAs regulate cancer onset and progression via Wnt/beta-catenin signaling pathway. Yonsei Med J. 2019:60(12):1117-28.

11. Xiao H, Liu M. Circular RNA hsa_circ_0053277 promotes the development of colorectal cancer by upregulating matrix metallopeptidase 14 via miR-2467-3p sequestration. J Cell Physiol. 2020;235(3):2881-90.

12. Zhang C, et al. Circular RNA hsa_circ_0006401 promotes proliferation and metastasis in colorectal carcinoma. Cell Death Dis. 2021;12(5):443.

13. Gao C, et al. Circ_0055625 knockdown inhibits tumorigenesis and improves radiosensitivity by regulating miR-338-3p/MSI1 axis in colon cancer. World J Surg Oncol. 2021;19(1):131.

14. Chu S, Wang H, Yu M. A putative molecular network associated with colon cancer metastasis constructed from microarray data. World J Surg Oncol. 2017;15(1):115

15. Fan $\mathrm{X}$, et al. Integrated analysis of RNA-binding proteins in human colorectal cancer. World J Surg Oncol. 2020;18(1):222.

16. Fang $Z$, et al. Identification of a prognostic gene signature of colon cancer using integrated bioinformatics analysis. World J Surg Oncol. 2021;19(1):13.

17. Wang $Y$, et al. Identification of a ferroptosis-related gene signature predictive model in colon cancer. World J Surg Oncol. 2021;19(1):135.

18. Gao M, et al. Comprehensive analyses of correlation and survival reveal informative IncRNA prognostic signatures in colon cancer. World J Surg Oncol. 2021:19(1):104.

19. Hansen TB, et al. Natural RNA circles function as efficient microRNA sponges. Nature. 2013;495(7441):384-8.

20. Zhang W, et al. Circ_DOCK1 regulates USP11 through miR-132-3p to control colorectal cancer progression. World J Surg Oncol. 2021;19(1):67.

21. Huang X, Lu S. MicroR-545 mediates colorectal cancer cells proliferation through up-regulating epidermal growth factor receptor expression in HOTAIR long non-coding RNA dependent. Mol Cell Biochem. 2017:431(1-2):45-54

22. Maziere P, Enright AJ. Prediction of microRNA targets. Drug Discov Today. 2007:12(11-12):452-8

23. Li K, et al. miR-378a-5p inhibits the proliferation of colorectal cancer cells by downregulating CDK1. World J Surg Oncol. 2021;19(1):54.

24. You W, et al. Downregulation of myosin VI reduced cell growth and increased apoptosis in human colorectal cancer. Acta Biochim Biophys Sin (Shanghai). 2016;48(5):430-6.

25. Wei AW, Li LF. Long non-coding RNA SOX21-AS1 sponges miR-145 to promote the tumorigenesis of colorectal cancer by targeting MYO6. Biomed Pharmacother. 2017;96:953-9. 
26. Chen LY, et al. NSD2 circular RNA promotes metastasis of colorectal cancer by targeting miR-199b-5p-mediated DDR1 and JAG1 signalling. J Pathol. 2019;248(1):103-15.

27. Li X, et al. Circular RNA circlTGA7 inhibits colorectal cancer growth and metastasis by modulating the Ras pathway and upregulating transcription of its host gene ITGA7. J Pathol. 2018;246(2):166-79.

28. Xu H, et al. CircRNA_0000392 promotes colorectal cancer progression through the miR-193a-5p/PIK3R3/AKT axis. J Exp Clin Cancer Res. 2020;39(1):283

29. Xu H, et al. RNA-Seq profiling of circular RNAs in human colorectal Cancer liver metastasis and the potential biomarkers. Mol Cancer. 2019;18(1):8

30. Liang Y, et al. Hsa_circ_0026416 promotes proliferation and migration in colorectal cancer via miR-346/NFIB axis. Cancer Cell Int. 2020;20:494

31. Pan Y, et al. Gene therapy for colorectal cancer by adenovirus-mediated siRNA targeting CD147 based on loss of the IGF2 imprinting system. Int J Oncol. 2015;47(5):1881-9.

32. Sousa $A R$, et al. Nanotechnology-based siRNA delivery strategies for metastatic colorectal cancer therapy. Int J Pharm. 2019;568:118530.

33. Ma $X$, et al. LnCRNA MCM3AP-AS1 upregulates CDK4 by sponging miR-545 to suppress $\mathrm{G} 1$ arrest in colorectal cancer. Cancer Manag Res. 2020;12:8117-24

34. Changjun L, et al. MiR-545-3p/MT1M axis regulates cell proliferation, invasion and migration in hepatocellular carcinoma. Biomed Pharmacother. 2018;108:347-54.
35. Chen S, et al. Downregulation of hsa_circ_0007580 inhibits non-small cell lung cancer tumorigenesis by reducing miR-545-3p sponging. Aging (Albany NY). 2020;12(14):14329-40.

36. Wang D, et al. MYO6 knockdown inhibits the growth and induces the apoptosis of prostate cancer cells by decreasing the phosphorylation of ERK1/2 and PRAS40. Oncol Rep. 2016;36(3):1285-92.

37. Lei $C$, et al. miR-143 and miR-145 inhibit gastric cancer cell migration and metastasis by suppressing MYO6. Cell Death Dis. 2017;8(10):e3101.

38. Yu H, et al. Lentivirus-mediated silencing of myosin VI inhibits proliferation and cell cycle progression in human lung cancer cells. Chem Biol Drug Des. 2015;86(4):606-13.

39. Liu Y, et al. Hsa_circ_0000231 knockdown inhibits the glycolysis and progression of colorectal cancer cells by regulating miR-502-5p/MYO6 axis. World J Surg Oncol. 2020;18(1):255.

40. Zarkavelis $\mathrm{G}$, et al. Current and future biomarkers in colorectal cancer. Ann Gastroenterol. 2017;30(6):613-21

\section{Publisher's Note}

Springer Nature remains neutral with regard to jurisdictional claims in published maps and institutional affiliations.
Ready to submit your research? Choose BMC and benefit from:

- fast, convenient online submission

- thorough peer review by experienced researchers in your field

- rapid publication on acceptance

- support for research data, including large and complex data types

- gold Open Access which fosters wider collaboration and increased citations

- maximum visibility for your research: over $100 \mathrm{M}$ website views per year

At BMC, research is always in progress.

Learn more biomedcentral.com/submissions 